\title{
The Passing of Ron Graham
}

\author{
Steve Butler
}

\section{October 31, 1935-July 6, 2020}

Ronald Lewis Graham, known to almost everyone as Ron, passed away at his home on the evening of July 6 . The cause of death was due to a progressive disease, atypical cystic fibrosis, that had been affecting him for years, but which in the last few months, then weeks and days caused an exponential decline in physical health. His mathematical abilities, however, never saw a decline. The day before, on July 5, Ron had reached out to several colleagues to talk about math and ongoing papers. Even on the morning of July 6, he was wanting to talk math, but was not physically able to carry a conversation.

Ron's first published papers, based on his dissertation in combinatorial number theory under the direction of Derrick H. Lehmer, appeared in 1963, though his mathematical explorations had started years earlier when he would carry out long computations (by hand!) of various mathematical problems. Immediately after graduation, he joined Bell Labs (later AT\&T Labs) where his mathematical interests, and output, broadened considerably. Over the course of his life, he had approximately 400 publications (with more yet to come) making fundamental contributions to most fields in combinatorics. His publications are maintained online at RonGraham.org.

At his passing, he also left behind hundreds of folders containing decades of computations, successful and failed attempts at problems, untapped ideas, and more. These folders will continue to be a source of new ideas for years to come, and so the mathematical contributions of Ron Graham will continue.

\section{Mathematical Influence}

In addition to his publications, Ron was a speaker in high demand who was well known for an ability to deliver talks which were both highly technical and entertaining. Moreover, his position at Bell Labs allowed him a high degree of support and flexibility which he was able to use to travel extensively. He soon became a central hub who was able to connect people and problems together, and was able to mentor and develop young mathematicians in combinatorics 
and beyond. He also served on the editorial boards of dozens of journals (simultaneously!). (And if that was not enough, in his spare time he was also active in juggling and trampolining.)

His research areas included the following.

- Ramsey theory, where with Bruce Rothschild and Joel Spencer, he literally wrote the book on the subject. He also popularized the notions of Ramsey theory through his eponymous Graham's number.

- Combinatorial number theory, the area of his dissertation, he continued producing results in this area right through to the end and had lifelong interests in Egyptian fractions and other number theory problems.

- Computational geometry, where his paper on the Graham scan for finding a convex hull of a set of points became one of the first papers in the field opening up the subject where hundreds of researchers followed, not so bad for a paper which he originally thought just a "throwaway" type result that would not be significant.

- Graph addressing. He described his paper with Henry Pollak (On embedding graphs in squashed cubes) as "one of my most fertile papers", which would later lead to investigations with László Lovász (Distance matrix polynomials of trees) on the distance matrix of a graph and show the graph theory community the importance of the distance matrix.

- Universal objects, which looked at ways to combine objects efficiently into a single structure, for example de Bruijn sequences. This would find applications in magic, which he worked on with Persi Diaconis. Their book Magical Mathematics would go on to win the Euler book prize.

- Quasirandom structures, a systematic way to see if a given constructed object exhibits behaviors associated with random objects. This initially started with graphs in a paper with Fan Chung and Richard Wilson (Quasirandom graphs), but grew to include hypergraphs, tournaments, set systems, and more.

- Combinatorial geometry. This included problems where identical objects (oftentimes circles, with Boris Lubachevsky, or squares, with Fan Chung or Paul Erdős) were to be packed as efficiently as possible into some large shape; counterintuitively, the optimal packings were not always the obvious arrangements.

- Steiner trees, which is a problem of looking for the shortest way to connect points via a network. His paper with Michael Garey and David Johnson (The complexity of computing Steiner minimal trees) showed this was NP-hard in general (though this did not stop Ron from continuing to work on special cases). This had some industrial motivation (billing for private long distance networks was based on minimum Steiner trees).

- Scheduling and bin packing. These are two sides to the same problem: arranging things in the most efficient way possible. His paper (Bounds for certain multiprocessing anomalies) was considered a landmark in theoretical computer science, as it contained, for the first time, a precise bound on the worst-case performance of a combinatorial algorithm. 
- Recreational mathematics. In a work with Joe Buhler, David Eisenbud, and Colin Wright (Juggling drops and descents), they were able to explore the mathematics of juggling, which he continued in additional papers and other coauthors. (The mathematics of juggling can also be described as the mathematics of discrete periodic patterns; but juggling sounds more fun.) This also ties in to his connections with Martin Gardner and his work in the popularization of mathematics.

The above list gives many of the major highlights, but is still not comprehensive. Ron's work also included algebraic combinatorics, circuit design, Apollonian circle packings, combinatorial games, card shuffling, probability, and more.

His scholarly contributions were recognized with being the first co-recipient of the Euler medal (ICA); the first co-recipient of the Polya Prize in Combinatorics (SIAM); the Steele Prize for Lifetime Achievement in 2003 (AMS); and being named a fellow for the ACM, AMS, and SIAM. He was also a member of the National Academy of Sciences as well as the Hungarian Academy of Sciences, in addition to being given six honorary degrees.

Over the course of his career, he wrote with over 200 different coauthors. But among them, his most important was Fan Chung, whom Ron married in 1983 and wrote over 100 papers together spanning the whole gamut of their collective interests. Their mathematical union was one of the most productive of the past 50 years.

\section{Reading the Book}

Ron was a long-time friend and confidant of Paul Erdős. They first met in person at a number theory conference in 1963, though they had already been communicating by mail by that time. In addition to their many publications (31, including the book Old and New Problems and Results in Combinatorial Number Theory), Ron also extensively helped manage Erdős' personal affairs, including acting as his informal accountant from 1973 through the time that Erdős left.

In the ledger that Ron kept for Erdős, the entry for September 20, 1996 reads: "EP leaves. Finally, he can read the Book." Now Ron and Erdös are able to read the Book together, undoubtedly with occasional pauses to declare "I knew it", or "What would happen if...".

\section{Note from the Editors-in-Chief}

Ron Graham was an esteemed member of the Editorial Board of Annals of Combinatorics ever since the journal was established. He was highly helpful and supportive and will be sorely missed.

Kolja Knauer, Matjaz Konvalinka, Marni Mishna

Publisher's Note Springer Nature remains neutral with regard to jurisdictional claims in published maps and institutional affiliations. 